\title{
SLIDING CONTROLLER DESIGN FOR THE GLOBAL CHAOS SYNCHRONIZATION OF IDENTICAL HYPERCHAOTIC YUJUN SYSTEMS
}

\author{
Sundarapandian Vaidyanathan ${ }^{1}$ \\ ${ }^{1}$ Research and Development Centre, Vel Tech Dr. RR \& Dr. SR Technical University \\ Avadi, Chennai-600 062, Tamil Nadu, INDIA \\ sundarvtuegmail.com
}

\begin{abstract}
This paper establishes new results for the sliding controller design for the global chaos synchronization of identical hyperchaotic Yujun systems (2010). Hyperchaotic systems are chaotic nonlinear systems having more than one positive Lyapunov exponent. Because of the complex dynamics properties of hyperchaotic system such as high capacity, high security and high efficiency, they are very useful in secure communication devices and data encryption. Using sliding mode control theory and Lyapunov stability theory, a general result has been obtained for the global chaos synchronization of identical chaotic nonlinear systems. As an application of this general result, this paper designs a sliding controller for the global chaos synchronization of hyperchaotic Yujun systems. Numerical results and simulations are shown to validate the proposed sliding controller design and demonstrate its effectiveness in achieving global chaos synchronization of hyperchaotic Yujun systems.
\end{abstract}

\section{KEYWORDS}

Hyperchaos, Synchronization, Sliding control theory, Lyapunov stability theory, Yujun system.

\section{INTRODUCTION}

Chaotic systems having more than one positive Lyapunov exponent are defined as hyperchaotic systems. Hyperchaotic systems have complex dynamics behaviour and exhibit special characteristics such as high capacity, high security and high efficiency.

The first hyperchaotic system was discovered by the German scientist, O.E. Rössler ([1], 1979). Some other classical hyperchaotic systems are hyperchaotic Lorenz-Haken system [2], hyperchaotic Chua's circuit [3], hyperchaotic Chen system [4], hyperchaotic Lü system [5], etc. Synchronization of chaotic systems occurs when two chaotic attractors are coupled together or when a chaotic attractor known as the master system drives another chaotic attractor known as the slave system. The goal of chaos synchronization is to device controllers so as to synchronize the states of the master and slave systems.

In the last two decades, there has been great attention devoted in the literature for the research on the global chaos and hyperchaos synchronization. Chaos synchronization has been applied in a wide variety of fields including physics [6], chemistry [7], ecology [8], secure communications [9-10], cardiology [11], neurology [12], robotics [13], etc.

DOI : 10.5121/ijait.2012.2402 
A seminal paper on chaotic synchronization was published by Pecora and Carroll [14], which paved the way for several new methods such as the active control method [15-16], adaptive control method [17-20], sampled-data feedback method [21], time-delay feedback method [22], backstepping method [23-24], sliding mode control method [25-26], etc.

This paper derives new results for the sliding controller design for the global chaos synchronization of identical hyperchaotic Yujun systems ([29], 2010). In robust control theory, sliding mode control is often practiced due to its inherent advantages such as easy realization, fast response and good transient performance as well as its insensitivity to parameter uncertainties and external disturbances.

This paper has been organized as follows. In Section 2, a main result has been proved for the design of a sliding mode controller for the global chaos synchronization of identical chaotic systems. In Section 3, the hyperchaotic Yujun system and its properties have been discussed. In Section 4, a sliding mode controller has been derived for the global chaos synchronization of identical hyperchaotic Yujun systems. Section 5 contains a summary of the main results obtained in this paper on the hyperchaotic Yujun systems.

\section{General Result for Sliding Controller Design}

In this section, we derive a general result for the sliding controller design for the global chaos synchronization of identical chaotic systems.

As the master or drive system, we take the chaotic system described by

$$
\dot{x}=A x+f(x)
$$

Here, $x \in R^{n}$ is the state of the system, $A$ is the $n \times n$ matrix of the system parameters and $f: R^{n} \rightarrow R^{n}$ is the nonlinear part of the system.

As the slave or response system, we take the controlled chaotic system described by

$$
\dot{y}=A y+f(y)+u
$$

Here, $y \in R^{n}$ is the state of the system and $u \in R^{m}$ is the control.

We define the synchronization error between the systems (1) and (2) as

$$
e=y-x
$$

It is easy to see that the error dynamics is given by

$$
\dot{e}=A e+\eta(x, y)+u,
$$

where

$$
\eta(x, y)=f(y)-f(x)
$$

The global chaos synchronization problem aims to find a controller $u$ such that 


$$
\lim _{t \rightarrow \infty}\|e(t)\|=0 \quad \text { for all } e(0) \in R^{n}
$$

For solving this synchronization problem, we first define the control $u$ as

$$
u=-\eta(x, y)+B v
$$

Here, $B$ is a constant column vector selected such that $(A, B)$ is controllable.

Substituting (6) into (4), the error dynamics simplifies to

$$
\dot{e}=A e+B v
$$

which is a linear time-invariant control system with single input $v$.

Thus, the original global chaos synchronization problem has been replaced by an equivalent control problem of stabilizing the equilibrium $e=0$ of the system (7) by a suitable choice of the sliding mode control.

In the sliding control design, we define the variable

$$
s(e)=C e=c_{1} e_{1}+c_{2} e_{2}+\cdots+c_{n} e_{n}
$$

where $C=\left[\begin{array}{llll}c_{1} & c_{2} & \cdots & c_{n}\end{array}\right]$ is a constant row vector to be determined.

In the sliding control design, we constrain the motion of the system (7) to the sliding manifold defined by

$$
S=\left\{e \in R^{n} \mid s(e)=0\right\}
$$

Here, $S$ is required to be invariant under the flow of the error dynamics (7).

When in sliding manifold $S$, the system (7) satisfies the following two conditions:

$$
s(e)=0
$$

which is the defining equation for the manifold $S$ and

$$
\dot{s}(e)=0
$$

which is the necessary condition for the state trajectory $e(t)$ of (7) to lie on the sliding manifold $S$.

From (7) and (8), the equation (10) can be rearranged as

$$
\dot{s}(e)=C[A e+B v]=0
$$

Solving (11) for the variable $v$, we obtain the equivalent control law 
International Journal of Advanced Information Technology (IJAIT) Vol. 2, No.4, August 2012

$$
v_{\mathrm{eq}}(t)=-(C B)^{-1} C A e(t)
$$

where $C$ is chosen such that

$$
C B \neq 0 .
$$

By substituting (12) into the error dynamics (7), we get the closed-loop error dynamics as

$$
\dot{e}=\left[I-B(C B)^{-1} C\right] A e
$$

The row vector $C$ is selected such that the system matrix of the system (13), i.e. $\left[I-B(C B)^{-1} C\right] A$, is Hurwitz. Then the system (13) is globally asymptotically stable.

To design the sliding controller for (7), we apply the constant plus proportional rate control law

$$
\dot{s}=-q \operatorname{sgn}(s)-k s
$$

where $\operatorname{sgn}(\cdot)$ denotes the sign function and the gains $q>0, k>0$ are found so that the sliding condition is satisfied and sliding motion will occur.

From equations (11) and (14), we can obtain the control $v(t)$ as

$$
v(t)=-(C B)^{-1}[C(k I+A) e+q \operatorname{sgn}(s)]
$$

i.e.

$$
v(t)= \begin{cases}-(C B)^{-1}[C(k I+A) e+q], & \text { if } s(e)>0 \\ -(C B)^{-1}[C(k I+A) e-q], & \text { if } s(e)<0\end{cases}
$$

Theorem 1. The master system (1) and the slave system (2) are globally and asymptotically synchronized for all initial conditions $x(0), y(0) \in R^{n}$ by the sliding mode control law

$$
u(t)=-\eta(x, y)+B v(t)
$$

where $v(t)$ is defined by the equation (15) and $B$ is a column vector chosen such that $(A, B)$ is completely controllable. Also, the gains $k$ and $q$ are positive.

Proof. By substituting the control laws (17) and (15) into the error dynamics (4), we get

$$
\dot{e}=A e-B(C B)^{-1}[C(k I+A) e+q \operatorname{sgn}(s)]
$$

We use Lyapunov stability theory to show that the error dynamics (18) is globally asymptotically stable.

For this purpose, we take the candidate Lyapunov function 


$$
V(e)=\frac{1}{2} s^{2}(e)
$$

which is a positive definite function on $R^{n}$.

By differentiating $V$ along the trajectories of (18) or the equivalent dynamics (14), we get

$$
\dot{V}(e)=s(e) \dot{s}(e)=-k s^{2}-q \operatorname{sgn}(s) s
$$

which is a negative definite function on $R^{n}$.

Hence, by Lyapunov stability theory [36], it is immediate that the error dynamics (18) is globally asymptotically stable for all initial conditions $e(0) \in R^{n}$.

\section{Sliding Controller Design for Global ChaOs SynChronization OF IDENTICAL HYPERCHAOTIC YUJUN SYSTEMS}

\subsection{Main Results}

In this section, we derive new results for the sliding controller design for the global chaos synchronization of identical hyperchaotic Yujun systems ([35], Yujun, 2010).

Thus, the master system is described by the hyperchaotic Yujun system

$$
\begin{aligned}
& \dot{x}_{1}=a\left(x_{2}-x_{1}\right)+x_{2} x_{3} \\
& \dot{x}_{2}=c x_{1}-x_{2}-x_{1} x_{3}+x_{4} \\
& \dot{x}_{3}=x_{1} x_{2}-b x_{3} \\
& \dot{x}_{4}=-x_{1} x_{3}+d x_{4}
\end{aligned}
$$

where $x_{1}, x_{2}, x_{3}, x_{4}$ are the states and $a, b, c, d$ are positive, constant parameters of the system.

The 4-D system (21) is hyperchaotic when $a=35, b=8 / 3, c=55$ and $d=1.5$.

Figure 1 illustrates the phase portrait of the hyperchaotic Yujun system. 
International Journal of Advanced Information Technology (IJAIT) Vol. 2, No.4, August 2012
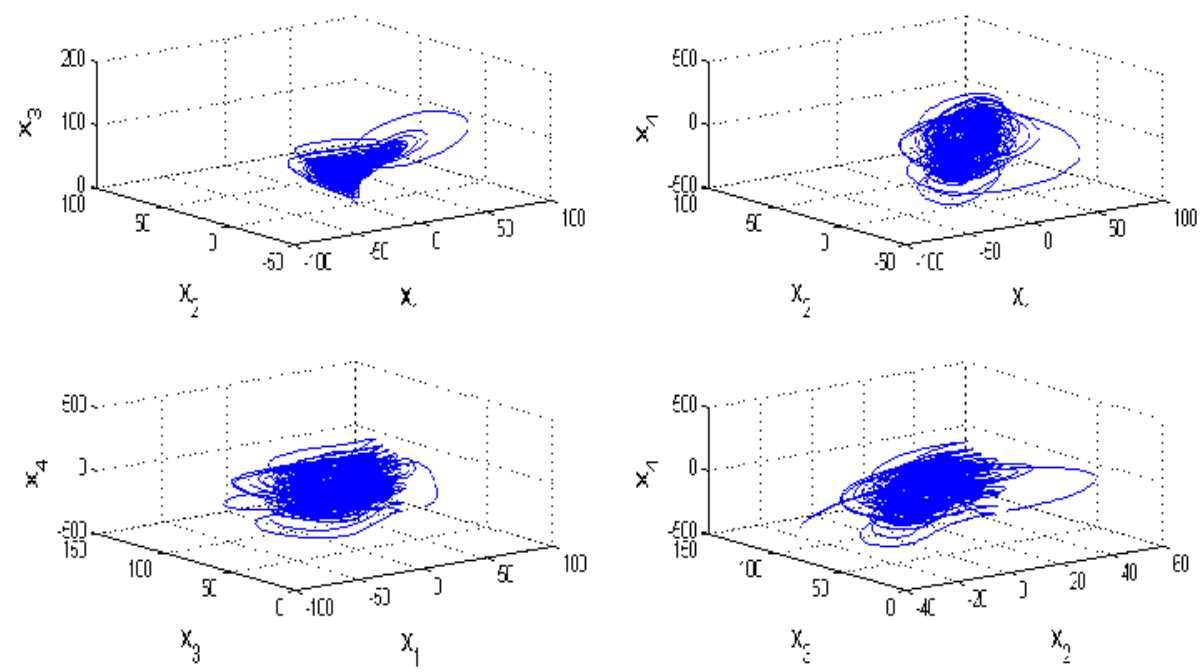

Figure 1. State Orbits of the Hyperchaotic Yujun System

The slave system is described by the controlled hyperchaotic Yujun system

$$
\begin{aligned}
& \dot{y}_{1}=a\left(y_{2}-y_{1}\right)+y_{2} y_{3}+u_{1} \\
& \dot{y}_{2}=c y_{1}-y_{2}-y_{1} y_{3}+y_{4}+u_{2} \\
& \dot{y}_{3}=y_{1} y_{2}-b y_{3}+u_{3} \\
& \dot{y}_{4}=-y_{1} y_{3}+d y_{4}+u_{4}
\end{aligned}
$$

where $y_{1}, y_{2}, y_{3}, y_{4}$ are the states and $u_{1}, u_{2}, u_{3}, u_{4}$ are the controllers to be designed.

The chaos synchronization error is defined by

$$
e_{i}=y_{i}-x_{i},(i=1,2,3,4)
$$

The error dynamics is easily obtained as

$$
\begin{aligned}
& \dot{e}_{1}=a\left(e_{2}-e_{1}\right)+y_{2} y_{3}-x_{2} x_{3}+u_{1} \\
& \dot{e}_{2}=c e_{1}-e_{2}+e_{4}-y_{1} y_{3}+x_{1} x_{3}+u_{2} \\
& \dot{e}_{3}=-b e_{3}+y_{1} y_{2}-x_{1} x_{2}+u_{3} \\
& \dot{e}_{4}=d e_{4}-y_{1} y_{3}+x_{1} x_{3}+u_{4}
\end{aligned}
$$

We write the error dynamics (24) in the matrix notation as

$$
\dot{e}=A e+\eta(x, y)+u
$$

where 
International Journal of Advanced Information Technology (IJAIT) Vol. 2, No.4, August 2012

$$
A=\left[\begin{array}{cccc}
-a & a & 0 & 0 \\
c & -1 & 0 & 1 \\
0 & 0 & -b & 0 \\
0 & 0 & 0 & d
\end{array}\right], \quad \eta(x, y)=\left[\begin{array}{c}
y_{2} y_{3}-x_{2} x_{3} \\
-y_{1} y_{3}+x_{1} x_{3} \\
y_{1} y_{2}-x_{1} x_{2} \\
-y_{1} y_{3}+x_{1} x_{3}
\end{array}\right] \text { and } u=\left[\begin{array}{l}
u_{1} \\
u_{2} \\
u_{3} \\
u_{4}
\end{array}\right]
$$

The sliding mode controller design is carried out as detailed in Section 2.

First, we set $u$ as

$$
u=-\eta(x, y)+B v
$$

where $B$ is chosen such that $(A, B)$ is controllable.

We take $B$ as

$$
B=\left[\begin{array}{l}
1 \\
1 \\
1 \\
1
\end{array}\right]
$$

The sliding mode variable is selected as

$$
s=C e=\left[\begin{array}{llll}
-1 & -2 & 0 & 1
\end{array}\right] e=-e_{1}-2 e_{2}+e_{4}
$$

which makes the sliding mode state equation asymptotically stable.

We choose the sliding mode gains as

$$
k=6 \text { and } q=0.2
$$

We note that a large value of $k$ can induce problems like chattering and hence a suitable value of $q$ is chosen to speed up the time taken to reach the sliding manifold and to reduce the system chattering.

From Eq. (15), we can obtain $v(t)$ as

$$
v(t)=-40.5 e_{1}-22.5 e_{2}+2.75 e_{4}+0.1 \operatorname{sgn}(s)
$$

Thus, the required sliding mode controller is obtained as

$$
u=-\eta(x, y)+B v
$$

where $\eta(x, y), B$ and $v(t)$ are given by (26), (28) and (30).

As an application of Theorem 1, we get the following result. 
International Journal of Advanced Information Technology (IJAIT) Vol. 2, No.4, August 2012

Theorem 2. The identical hyperchaotic Yujun systems given by the equations (21) and (22) are globally and asymptotically synchronized for all initial conditions with the sliding mode controller u defined by the equation (31).

\subsection{Numerical Results}

For numerical simulations, the fourth-order Runge-Kutta method with time-step $h=10^{-6}$ is used to solve the hyperchaotic Yujun systems (21) and (22) with the sliding mode controller $u$ given by (31) using MATLAB.

In the hyperchaotic case, the parameter values are given by

$$
a=35, b=8 / 3, c=55, d=1.5
$$

The sliding mode gains are chosen as $k=6$ and $q=0.2$

The initial values of the master system (21) are taken as

$$
x_{1}(0)=5, x_{2}(0)=22, x_{3}(0)=-10, x_{4}(0)=-12
$$

and the initial values of the slave system (22) are taken as

$$
y_{1}(0)=10, y_{2}(0)=-6, y_{3}(0)=21, y_{4}(0)=6
$$

Figure 2 illustrates the complete synchronization of the identical hyperchaotic Yujun systems (21) and (22). Figure 3 shows time history of the synchronization errors $e_{1}(t), e_{2}(t), e_{3}(t), e_{4}(t)$.
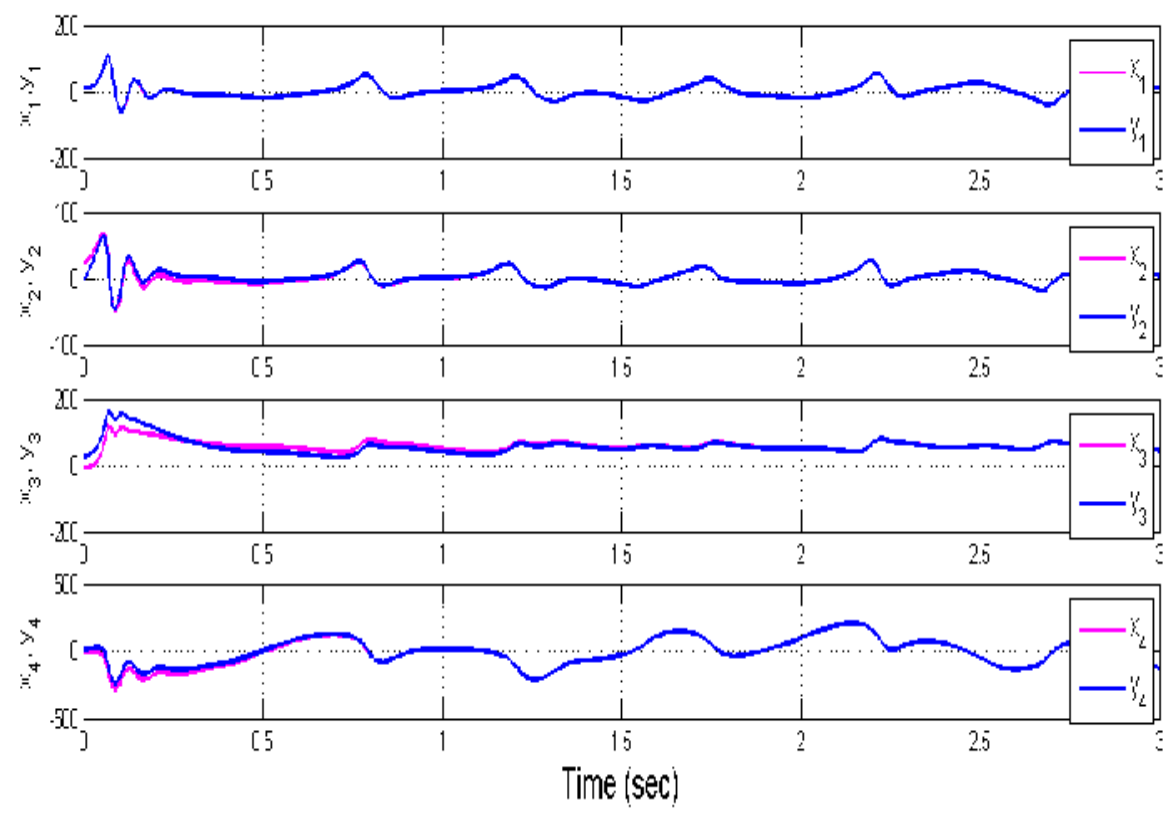

Figure 2. Complete Synchronization of Identical Hyperchaotic Yujun Systems 


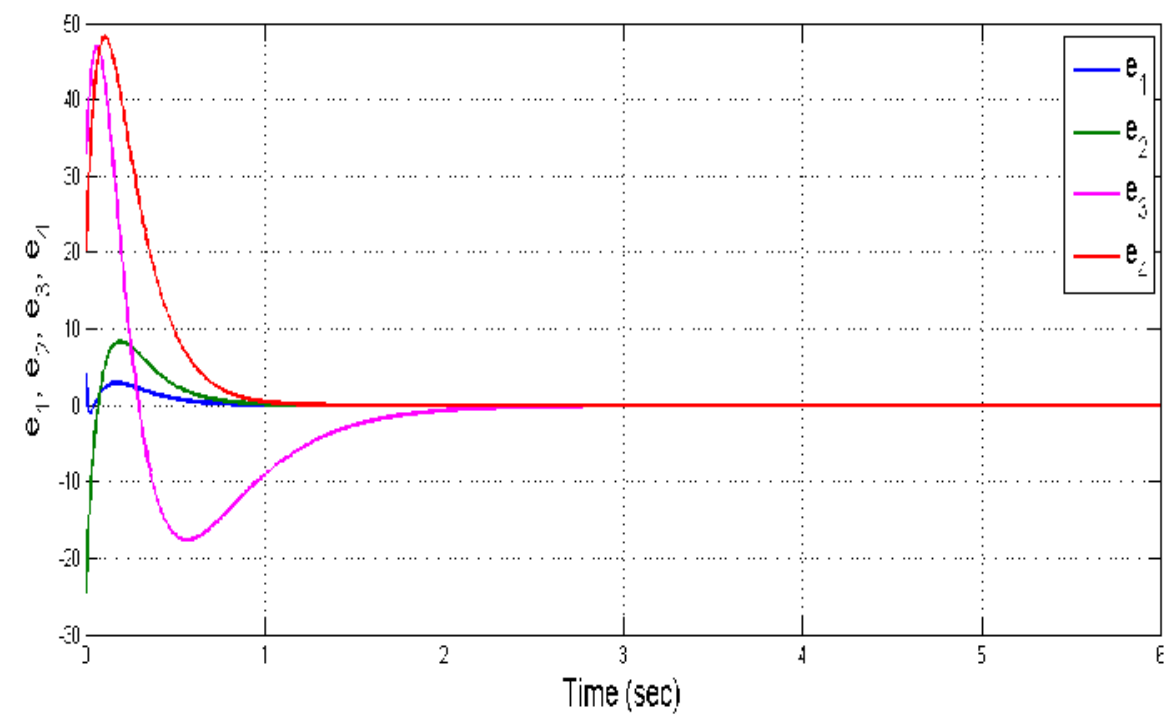

Figure 3. Time Responses of Error States - $e_{1}(t), e_{2}(t), e_{3}(t), e_{4}(t)$

\section{Conclusions}

In this paper, we derived new results for the sliding controller design for the global chaos synchronization of identical hyperchaotic Yujun systems (2010). Our synchronization results have been proved using the sliding mode control theory and Lyapunov stability theory. Numerical results and figures have been depicted to validate and illustrate the effectiveness of the sliding controller design for the global synchronization of hyperchaotic Yujun systems.

\section{REFERENCES}

[1] Rössler, O.E. (1979) “An equation for hyperchaos,” Physics Letters A, Vol. 71, pp 155-157.

[2] Ning, C.Z. \& Haken, H. (1990) "Detuned lasers and the complex Lorenz equations: Subcritical and supercritical Hopf bifurcations," Physical Review A, Vol. 41, pp 3826-3837.

[3] Kapitaniak, T. \& Chua, L.O. (1994) "Hyperchaotic attractor of unidirectionally coupled Chua's circuit," International Journal of Bifurcation \& Chaos, Vol. 4, pp 477-482.

[4] Li, Y.X., Tang, W.K.S. \& Chen, G. (2005) "Generating hyperchaos via state feedback control," International Journal of Bifurcation and Chaos, Vol. 10, pp 3367-3375.

[5] Chen, A., Lu, J., Lü, Yu, S. (2006) "Generating hyperchaotic Lü attractor via state feedback control," Physica A, Vol. 364, pp 103-110.

[6] Lakshmanan, M. \& Murali, K. (1996) Nonlinear Oscillators: Controlling and Synchronization, World Scientific, Singapore.

[7] Han, S.K., Kerrer, C. \& Kuramoto, Y. (1995) "Dephasing and bursting in coupled neural oscillators", Phys. Rev. Lett., Vol. 75, pp 3190-3193.

[8] Blasius, B., Huppert, A. \& Stone, L. (1999) "Complex dynamics and phase synchronization in spatially extended ecological system”, Nature, Vol. 399, pp 354-359.

[9] Feki, M. (2003) "An adaptive chaos synchronization scheme applied to secure communication", Chaos, Solitons and Fractals, Vol. 18, pp 141-148.

[10] Murali, K. \& Lakshmanan, M. (1998) "Secure communication using a compound signal from generalized synchronizable chaotic systems", Phys. Rev. Lett. A, Vol. 241, pp 303-310.

[11] Garfinkel, A., Spano, M.L., Ditto, WL. \& Weiss, J.A., (1992) "Controlling cardiac chaos", Science, Vol. 257, 1230-1235. 
International Journal of Advanced Information Technology (IJAIT) Vol. 2, No.4, August 2012

[12] Shelhamer, M (2005) Nonlinear Dynamics in Physiology: A State-Space Approach, World Scientific Publishing, Singapore.

[13] Nakamura, Y. \& Sekiguchi, A. (2001) "Chaotic mobile robot", IEEE Transactions on Robotics and Automation, Vol. 17, pp 898-904.

[14] Pecora, L.M. \& Carroll, T.L. (1990) "Synchronization in chaotic systems", Phys. Rev. Lett., Vol. 64, pp 821-824.

[15] Ho, M.C. \& Hung, Y.C. (2002) "Synchronization of two different chaotic systems by using generalized active control", Physics Letters A, Vol. 301, pp 424-428.

[16] Huang, L., Feng, R. \& Wang, M. (2004) "Synchronization of chaotic systems via nonlinear control”, Physics Letters A, Vol. 320, pp 271-275.

[17] Liao, T.L. \& Tsai, S.H. (2000) "Adaptive synchronization of chaotic systems and its applications to secure communications”, Chaos, Solitons and Fractals, Vol. 11, pp 1387-1396.

[18] Park, J.H. (2007) "Adaptive controller design for modified projective synchronization of GenesioTesi chaotic system with uncertain parameters", Chaos, Solitons \& Fractals, Vol. 34, pp 11541159.

[19] Sundarapandian, V. (2011) "Adaptive control and synchronization of hyperchaotic Chen system", International Journal of Information Technology Convergence and Services, Vol. 1, No. 3, pp 2233.

[20] Sundarapandian, V. (2011) "Adaptive synchronization of hyperchaotic Lorenz and hyperchaotic Lü systems”, International Journal of Instrumentation and Control Systems, Vol. 1, pp 1-18.

[21] Zhao, J. \& Lü, J. (2006) "Using sampled-data feedback control and linear feedback synchronization in a new hyperchaotic system," Chaos, Solitons \& Fractals, Vol. 35, pp. 376-382.

[22] Park, J.H. \& Kwon, O.M. (2003) "A novel criterion for delayed feedback control of time-delay chaotic systems", Chaos, Solitons and Fractals, Vol. 17, pp 709-716.

[23] Yu, Y.G. \& Zhang, S.C. (2006) "Adaptive backstepping synchronization of uncertain chaotic systems”, Chaos, Solitons and Fractals, Vol. 27, pp 1369-1375.

[24] Suresh, R. \& Sundarapandian, V. (2012) "Global chaos synchronization of Windmi and Coullet chaotic systems by backstepping control”, Far East Journal of Mathematical Sciences, Vol. 67, No. 2, pp 265-282.

[25] Konishi, K., Hirai, M. \& Kokame, H. (1998) "Sliding mode control for a class of chaotic systems", Physics Letters A, Vol. 245, pp 511-517.

[26] Sundarapandian, V. (2011) "Hybrid chaos synchronization of hyperchaotic Newton-Leipnik systems by sliding mode control", International Journal of Control Theory \& Computer Modelling, Vol. 1, No.2, pp 1-10.

[27] Yujun, N. Xingyuan, W., Mingjun, W. \& Huaguang, Z. (2010) "A new hyperchaotic system and its circuit implementation", Communications in Nonlinear Science and Numerical Simulation, Vol. 15, pp 3518-3524.

[28] Hahn, W. (1967) The Stability of Motion, Springer, New York.

\section{Author}

Dr. V. Sundarapandian earned his Doctor of Science degree in Electrical and Systems Engineering from Washington University, Saintü Louis, USA in May 1996. He is a Professor at the R \& D Centre at Vel Tech Dr. RR \& Dr. SR Technical University, Chennai, Tamil Nadu, India. He has published over 275 refereed papers in international journals. He has published over 170 papers in National and International Conferences. He is the Editor-in-Chief of the AIRCC Journals - International Journal of Instrumentation and Control Systems, International Journal of Control Systems and Computer Modelling, International Journal of Information Technology, Control and Automation, International

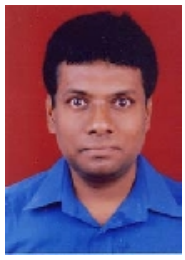
Journal of Chaos, Control, Modelling and Simulation and International Journal of Information Technology, Modeling and Computing. His research interests are Linear and Nonlinear Control Systems, Chaos Theory and Control, Soft Computing, Optimal Control, Operations Research, Mathematical Modelling and Scientific Computing. He has delivered several Key Note Lectures on Control Systems, Chaos Theory and Control, Operations Research, Mathematical Modelling, and Scientific Computing using MATLAB and SCILAB. 